\title{
Proper Motion study of globular cluster NGC 4590
}

\author{
Gaurav Singh, Ramakant Singh Yadav* \\ Aryabhatta Research Institute of observational sciencES (ARIES), \\ Manora Peak Nainital 263001, India
}

\begin{abstract}
The similar age of the stars in star cluster system makes them an interesting objects for the study of stellar evolution. However, for such studies, it is essential to reduce the field star contamination. Proper motion of the stars in the region of star clusters provides a unique possibility of getting membership information of the stars. The mean absolute proper motion of the globular cluster NGC 4590 is derived as $-2.95 \pm 0.54 \mathrm{mas} \mathrm{yr}^{-1}$ and $2.03 \pm 0.52 \mathrm{mas} \mathrm{yr}^{-1}$ along right ascension (RA) and declination (DEC) respectively using $\approx 11,000$ stars having an error less than 7 mas $_{\mathrm{yr}^{-1}}$. We propose a similar study of globular clusters with the CCD imager mounted on the $3.6 \mathrm{~m}$ Devasthal Optical telescope (DOT).
\end{abstract}

\section{Introduction}

Galactic globular clusters play an important role to explore the extent, formation, and evolution of our Galaxy. Proper motions (PMs) of the stars provide velocity information in two orthogonal directions (Sagar Bhatt 1989) and enable us to determine membership information of the stars in the clusters. The results of the Hipparcos mission offer new possibilities for the determination of absolute PMs of galactic globular clusters and other objects of interest for galactic kinematics. The use of Hipparcos reference stars offer a unique possibility for the determination of absolute PMs at low galactic latitudes without suitable extragalactic background objects.

M68/NGC 4590 is a galactic globular cluster located in halo region of our galaxy $\left(\alpha_{2000}=12^{h}\right.$ $39^{m} 27^{s} .98, \delta_{2000}=-26^{h} 44^{m} 38^{s} .6 ; l=299^{\circ} .63, b=36^{\circ} .05$; Harris 1996). Dinescu et al. (1999) measured the absolute PMs of NGC 4590 with photographic plates. The authors found $\mu_{\alpha} \cos \delta=$

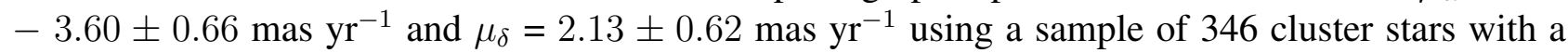
photographic magnitude below $V \approx 16$ and background galaxies as a reference.

The main purpose of the present study is to determine accurate absolute PMs for the stars brighter than $V \approx 20 \mathrm{mag}$ in the field of NGC 4590. The observations used for this study, their astrometric reduction and calibration procedures are described in Sect. 2 and in Sect. 3 we discuss the results using color-magnitude diagrams (CMDs) and vector point diagrams (VPDs) of the cluster. Finally in Sect. 4, we discuss the results.

\footnotetext{
*Corresponding author, e-mail: rkant@aries.res.in
} 
Table 1: Description of the WFI 2.2-m telescope data used for our study.

\begin{tabular}{|l|clc|}
\hline Epoch & Filters & $\begin{array}{l}\text { Exposure Time } \\
(\mathrm{sec})\end{array}$ & Airmass \\
\hline First & $B$ & $3 \times 180$ & 1.2 \\
$19 / 02 / 2002$ & $V$ & $3 \times 180$ & 1.2 \\
\hline Second & $B$ & $1 \times 10,1 \times 30$ & 1.1 \\
$02 / 04 / 2014$ & $V$ & $1 \times 10,1 \times 30$ & 1.1 \\
\hline
\end{tabular}

\section{Data}

The images used in this work were acquired with the 2.2-m ESO/MPI telescope located at La Silla, Chile. The WFI@2.2-m consists of eight $2048 \times 4096$ EEV CCDs with $0^{\prime \prime} .238$ pixel $^{-1}$ resulting in a total field-of-view of $34^{\prime} \times 33^{\prime}$.

Our PM study of the stars in the cluster region was done by using archive WFI@2.2-m filter images that were taken on February 19, 2002 (first epoch) and on April 02, 2014 (second epoch). The first epoch data consists of three images in $B$ and $V$ filters. While two images in $B$ and $V$ filters were used as the second epoch data. The log of the observations used is given in Table 1.

\subsection{Astrometric and photometric reductions}

The reduction procedures described in Anderson et al. (2006) were adopted for the WFI@2.2-m CCD images. This includes de-biasing, flat-fielding and correction for cosmic rays. To avoid the spatial variability of the point spread function (PSF), an array of 15 empirical PSFs per CCD chip ( 3 across and 5 high) was constructed to obtain the position and fluxes of the stars. An iterative procedure was designed to work with the brightest down to the faintest stars and find their precise position and instrumental flux for the $B$ and $V$ exposures. In the last step, we apply a local transformation approach to measure the motion with respect to field stars. We therefore pick a particular population, set its motion to zero and measure the motions relative to that population. Since cluster stars tend to have less internal dispersion than field stars, it is natural to use the cluster stars as the reference. The obvious cluster-member stars are identified either by their location in the CMD, or iteratively by the motion itself, or both. They are used as the basis for the transformation.

\subsection{Astrometric and photometric calibrations}

In order to transform the instrumental $B$ and $V$ magnitudes into the standard Johnson-Cousin system, we used a list of secondary standard stars provided by Stetson (http://cadcwww.hia.nrc.ca/standards/). The next step is to transform the pixel coordinates $X$ and $Y$ into right ascension (RA) and declination (Dec). To transform the averaged $X$ and $Y$ coordinates into RA and Dec of J2000, we used the online digitized sky ESO catalog in the sky cat software as an absolute astrometric reference frame (http://archive.eso.org/cms/tools-documentation/skycat.html).

\section{Results and discussions}

A total of three images in the first epoch and two images in the second epoch were used to determine PMs. A set of probable cluster members was initially selected using the $V$ versus $(B-V) \mathrm{CMD}$. The 
selected stars are located on the cluster sequences in the CMD and have a magnitude range of $12 \leq V$ $\leq 20$. They are used as a local reference frame to transform the coordinates of the stars between the epochs.

In Fig. 1 we show how effectively the proper motions separate the field stars by using VPDs in the top panels and $V$ versus ( $B-V$ ) CMDs in the bottom panels. The left panel shows the whole sample of stars, while the middle and right panel show probable cluster members and field stars, respectively. The VPD in the middle panel has a circle of radius $\approx 7$ mas/yr around the cluster centroid, which is provisionally our criterion for discriminating between members (inside) and field stars (outside).

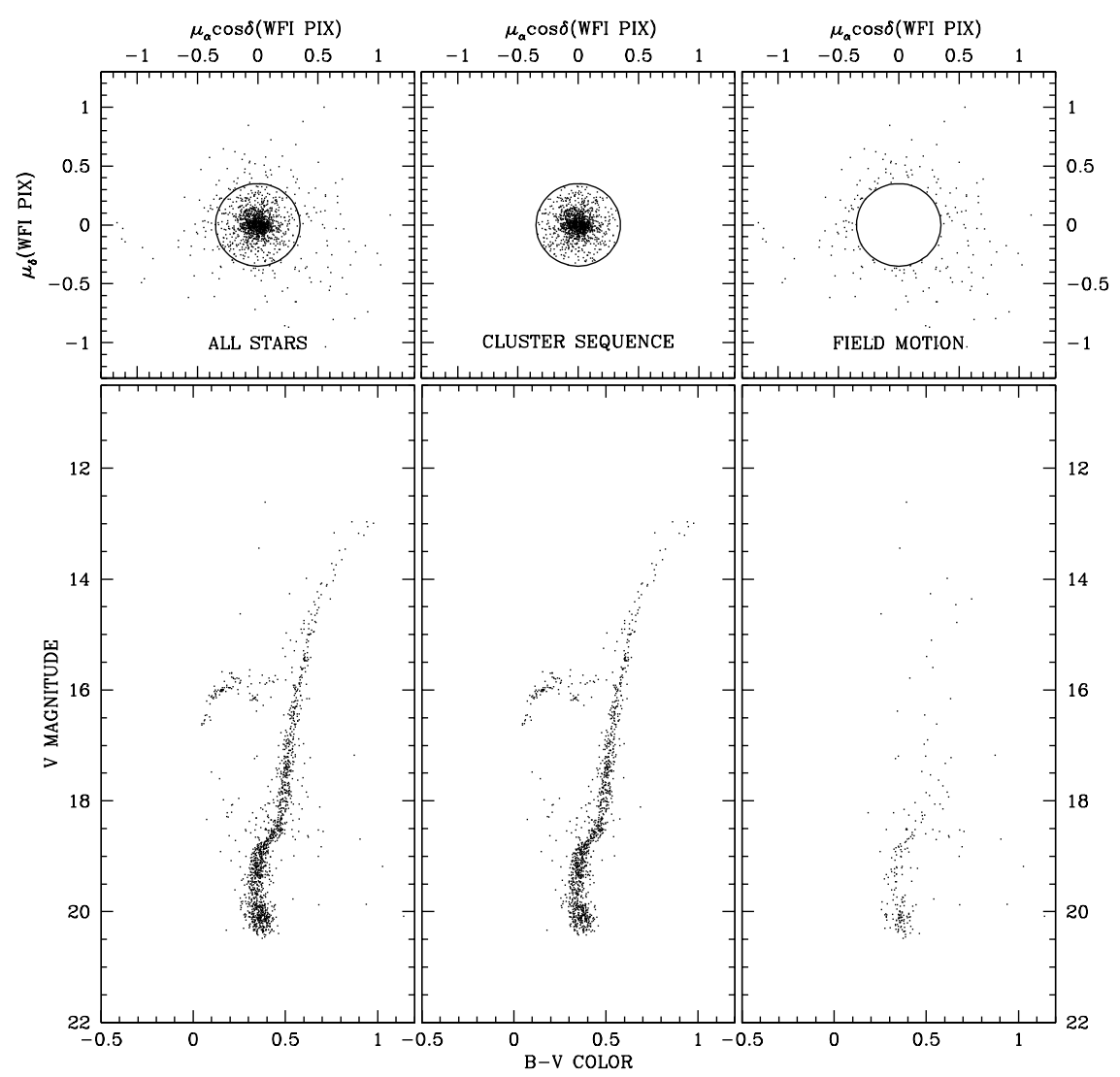

Figure 1: Top panels: Proper motion vector point diagram. Bottom panels: $V$ versus $(B-V)$ color-magnitude diagram.

The SPM 4.0 catalog (Dinescu et al. 2011) lists the absolute proper motions for some stars in the region of NGC 4590. We found 262 common stars with the SPM 4.0 catalog brighter than $V=$ 17.92 mag. We estimated the absolute PM of these stars by comparing our catalog with the SPM 4.0 catalog. The results of the comparison are shown in Fig. 2.

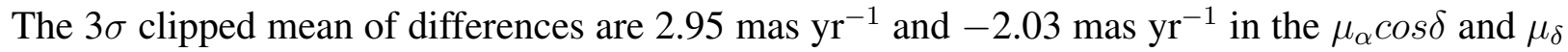
directions, respectively. In Fig. 3, we show VPD of the absolute PM. The mean value of the absolute

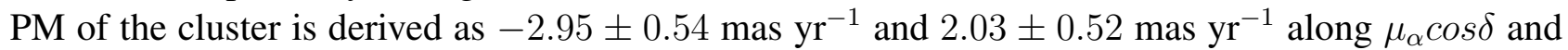
$\mu_{\delta}$, respectively. The absolute proper motion derived using astrometric procedures is similar to those

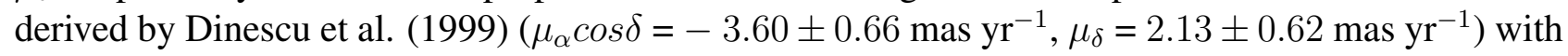
photographic plates. 


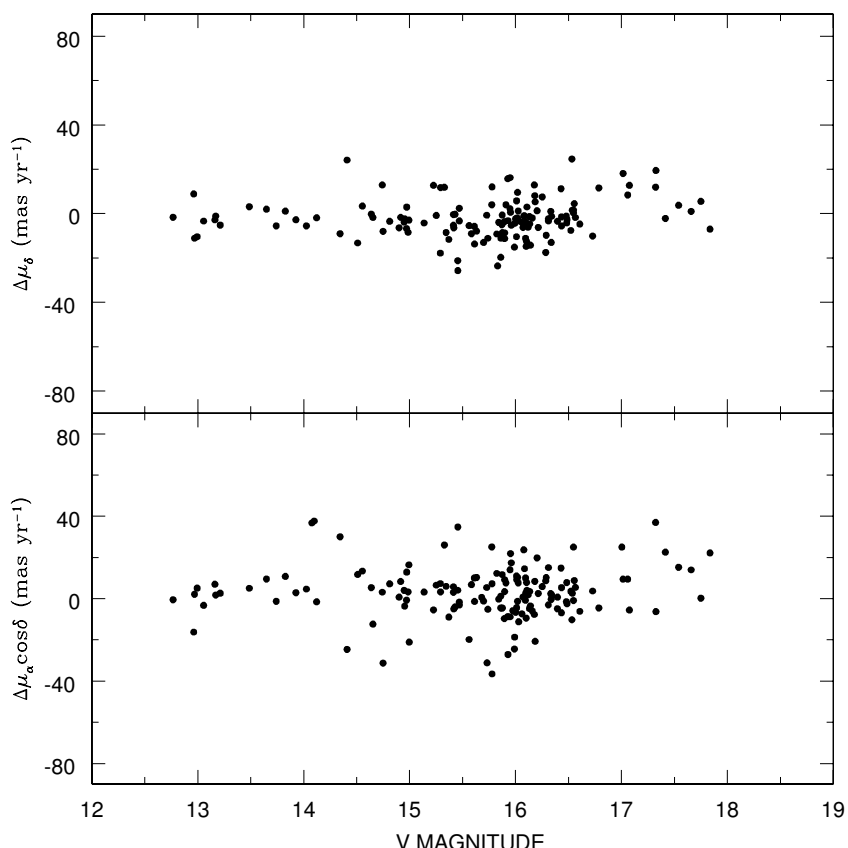

Figure 2: Overview of the proper motion difference between the SPM 4.0 catalog and our data.

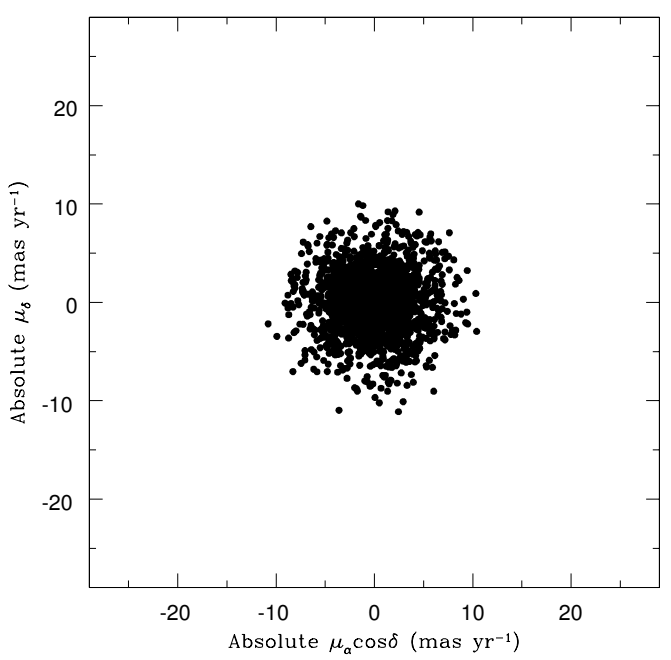

Figure 3: vector point diagram of the absolute proper motion.

\section{Summary and Conclusions}

We demonstrate that CCD observations taken just 12 years apart can provide good PMs to further study the cluster. The mean absolute PMs in the region of the globular cluster NGC 4590 were found

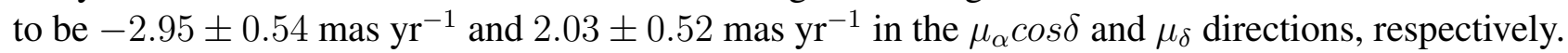
They were derived by using 11,000 stars brighter than $V=20$ mag.

This data is very useful in assigning the membership probabilities of blue stragglers and many variable stars in the cluster region. We propose a similar study of globular clusters with CCD imager mounted on the 3.6-m DOT.

\section{Acknowledgements}

We would like to acknowledge ESO, based on observations made with ESO Telescopes at the La Silla Observatory under program ID 68.D-0265(A). This research used the facilities of the Canadian Astronomy Data Centre operated by the National Research Council of Canada with the support of the Canadian Space Agency. This research has made use of the VizieR catalog to access tool, CDS, Strasbourg, France.

\section{References}

Anderson J., Bedin L. R., Piotto G., Yadav R. K. S., Bellini A. 2006, A\&A, 454, 1029

Dinescu D. I., van Altena W. F., Girard T. M., Lopez C. E. 1999, AJ, 117, 277

Harris W. E. 1996, AJ, 2013, 146, 119

Sagar R., Bhatt H. C. 1989, MNRAS, 236, 865 